Relmecs, junio 2017, vol. 7, no. 1, e016, ISSN 1853-7863

Universidad Nacional de La Plata. Facultad de Humanidades y Ciencias de la Educación. Centro Interdisciplinario de Metodología de las Ciencias Sociales.

Red Latinoamericana de Metodología de las Ciencias Sociales

\title{
Metodología: reflexión y creatividad frente a las recetas
}

\author{
The scope of a community diagnosis on Suicide: Reflections from \\ experience
}

Héctor Mora Nawrath *

* Facultad de Ciencias Sociales. Universidad Católica de Temuco, Chile |

Para iniciar esta presentación, me parece pertinente recuperar algunas reflexiones que introduce Charles Ragin en el prefacio de la obra The Comparative Method. Moving Beyond Qualitative and Quantitative Strategies publicada en 1987폴 trabajo del que trata unos de los artículos de este volumen. Ragin señala que su interés en la formalización de técnicas se encuentra en la frustración que se desprende de su entrenamiento en determinados tipos de análisis y la imposibilidad para dar respuesta a ciertos problemas asociados, para su caso, a la comparación por la vía estadística tradicional. Podríamos plantear que Rangin se "indisciplinó", debido a que a través de una interrogación profunda sobre los supuestos de ciertos métodos y sobre las convenciones en materia de análisis de datos abandonó las prescripciones que a modo de recetas se suelen repetir mecánicamente para buscar creativamente una solución. Tomó un producto de la reflexión matemática, el álgebra booleana, y la pensó como dispositivo para el análisis social. El método comparativo cualitativo fue una respuesta creativa: un ensamblaje técnico que cobró sentido a la luz de un sustento epistemológico. Al mismo tiempo, desnaturalizó las parcelaciones y sorteó los obstáculos, tan comunes en dicha época, que hacían, por ejemplo, inconmensurable lo "cualitativo” y lo “cuantitativo”. Por cierto, aún no es raro que a más de uno le cause extrañeza proponer un "análisis comparativo de casos cualitativos" utilizando el álgebra booleana: ¡la matemática no es cualitativa! o ¡el estudio de casos no tiene que ver con matemáticas!, podrá advertir, ¡es otro paradigma!

Podríamos afirmar que este tipo de reflexiones han estado en el origen de la mayoría -si no de la totalidadde los métodos de investigación social -estos ensambles epistemológico-técnicos-. Si entendemos un método como el producto de un proceso creativo, ello tiene que ver con que en su origen respondió al interrogante de ¿cómo analizar o abordar tal clase de fenómenos o tal problemática?, donde esta interrogante no sólo implica un desafío cognoscitivo sino también práctico. 
Por tanto, un método de investigación puede ser definido en la articulación de diversas técnicas (recolección, calidad, selección de unidades, análisis) en el marco de ciertas claves teórico-epistemológicas, lo que da lugar a una nueva estructura de sentido que permite abordar determinados problemas de conocimiento. El método tiene su origen en una o varias disciplinas y expresa la creatividad de quienes contribuyen a su desarrollo, al lograr vincular técnicas o herramientas que han sido propuestas en diversos campos, incluso fuera de la disciplina y de la ciencia que lo categorizan (ciencia social/ciencia natural).

A cuenta de lo que propone Edgar Morin, podríamos agregar que todo método se constituye en la dinámica prescriptiva/descriptiva; al definir su contenido fija los fundamentos y lineamientos generales. Un método es instituido y, a través de dicho acto, apropiado, socializado -vía cátedras y manuales- y corporizado; incluso puede nutrir narrativas que definen identidades y demarcar territorios disciplinarios o académicos. Por otro lado, al observar su trayectoria a la luz de la puesta en marcha en investigaciones concretas, emerge la dimensión descriptiva o redescriptiva que se recoge en la experiencia de cara a la adecuación y con ella a la dinamización en una investigación concreta. Ello expresa, como dirían Bourdieu y Wacquant (2005 [1992]), una reflexión sobre los métodos en su uso real en el trabajo científico, lo que se diferenciaría del cultivo del método por el método mismo, abstraído de todo contexto de aplicación -lo que derivaría en el metodologísmo- y condenado a reproducir las recetas o reglas "para cualquier objeto" sin indagar sobre las condiciones de producción, los supuestos y los efectos que dichas prescripciones generan.

La dinámica prescriptiva/descriptiva es la que hace posible la reinterpretación o lectura creativa de cada método; recoge la experiencia y además se alimenta del debate epistemológico de cada época. Pero, como afirmará Sánchez Carrión (2001) a propósito del análisis exploratorio de datos de Tukey, su aceptación y popularidad -la innovación- no dependerá exclusivamente de la originalidad y consistencia de la propuesta, ya que se distinguen de esta manera una naturaleza cognitiva (que el invento sea potencialmente inventable) y otra social (que lo inventado se acepte masivamente y que sea útil para determinados grupos sociales).

Desde una aproximación genealógica -como una metáfora del parentesco- es posible observar el conjunto de alteraciones creativas -por las uniones a partir de las cuales se puede trazar la filiación y distinguir entre parientes y no parientes- que un método ha experimentado desde que fue enunciado, y de este modo comprender cómo se inscribe en la historia de las ideas y en la historia de la ciencia. En esta perspectiva, entre las múltiples fuentes que pueden dar origen a un método -esfuerzos históricos desde distintas disciplinas-, es posible citar el libro El desarrollo de del análisis de redes sociales, publicado en 2005 por Linton Freeman, en el cual se demuestra cómo la formalización de este análisis implicó la incorporación de una serie de aportes, en tiempos y desde prácticas disciplinares diversas: matemática, psicología social, antropología, sociología, informática. Algo similar ha pasado con las etnografías y los análisis del discurso.

Podríamos concluir señalando que las re-interpretaciones de un método (definidas en tiempo y espacio) pueden ser entendidas como estilos a la luz de lo que Ricoeur (2008) denomina exceso de sentido, y que se define como aquello que complementa, en un único movimiento, una significación secundaria gracias a una significación primaria o literal que se desprende de la comprensión de nuevos modos de ser. Un estilo en tanto variación debe su condición estilística a lo que se añade o agrega al "texto original”, y enriquece la interpretación desde una determinada tradición/posición. Esto cabe, por ejemplo, para métodos de análisis textual (las variantes del análisis del discurso y del contenido), participativos, las etnografías, los métodos de redes, etc. Según esta argumentación sería más pertinente hablar de estilos, lo que aporta matices respecto de una totalidad homogénea e invariante ${ }^{2}$. Un estilo, a su vez, permite diferenciar de otras propuestas y a la vez reconocer ciertas continuidades y conexiones.

Varios de los trabajos que componen este volumen pueden ser pensados como contribuciones a lo señalado con anterioridad. 
La reflexión que Galati lleva a cabo en el artículo "El pensamiento complejo y transdisciplinario como marco de investigación científica” se sitúa desde el "indisciplinamiento", lo que implica jugar con las fronteras que imponen las disciplinas y la ciencia convencional para pensar la "realidad social” y la ciencia a través de un abordaje transdisciplinario que se nutre con los fundamentos de la complejidad. El trabajo constituye un aporte porque articula una reflexión metodológica referida a cómo hacer investigación desde las claves complejas y transdisciplinarias, y sitúa como posibilidad la discutida aproximación multimétodo y la triangulación. La propuesta releva como un aspecto importante la creatividad, ello frente a la metodologización que encierra la investigación que define a los métodos como recetas. El trabajo de Galati nos impulsa a pensar tanto los límites y la posibilidad de las categorías que en él se articulan como a revisar nuestras perspectivas y disposiciones al disciplinamiento que se reproduce en la formación de nuevos científicos sociales.

Le siguen trabajos que ilustran la puesta en escena de distintas propuestas de análisis, y que comparten como idea central visibilizar o explicitar los recursos desplegados en la construcción del objeto.

El trabajo “Análisis comparativo cualitativo como herramienta analítica”, presentado por Rosati y Chazarreta, nos muestra la formalización de la creatividad. Tras una fundamentación epistemológica que sitúa el sentido de lo "cualitativo" a un nivel distinto al del paradigma o al del método, el artículo nos lleva a un plano técnico ligado al análisis para mostrar en términos prácticos la aplicación de un tipo de análisis disponible que podemos situar en la familia de los análisis de datos categóricos. Es la propuesta -ya comentada- de Charles Ragin que los autores aplican a dos casos concretos: uno vinculado al trabajo de recolección realizada por de los algodoneros de la provincia del Chaco, y otro, a la caracterización de las empresas vitivinícolas de Mendoza.

Presentan cuestiones asociadas a los "rudimentos” del álgebra booleana y a su operativización en el análisis, para luego, a través de un modelo de análisis, explorar la relación entre variables asociadas al trabajador (nivel educativo, movilidad laboral, experiencia en operación de tractores y año de ingreso al mercado laboral) que se vinculan a la técnica de cosecha, y otras, que considera variables -año de inicio en la actividad, origen del capital, orientación del mercado, cantidad de vino elaborado y tipo de productos-, a partir de las cuales construir una tipología.

Como ya señalamos, el trabajo de Ragin fue creativo al vincular -a raíz de ciertos interrogantes- elementos ya disponibles en una forma innovadora. Si bien puede parecer una receta -pues hay un formalismo presentees un aporte, en tanto herramienta, disponible para utilizar frente a determinados problemas. Los casos ilustrados por Rosati y Chazarreta, considero, van en esta línea.

Ernesto Meccia presenta la aplicación de una de las variantes del análisis del discurso -que refiere como análisis socio-lingüístico- que le permiten ir articulando una propuesta de análisis al recuperar aportes de Foucault, Charaudeau y Mingueneau, Ducrot, entre otros. El trabajo se sitúa en el periodo del actual gobierno de Argentina, y tomo como referencia los discursos sobre inmigración que se han evacuado a través tres programas de televisión, en emisiones que se ubican entre octubre y noviembre de 2016. El trabajo tiene como centro a "los recursos lingüísticos" que movilizan los enunciadores y que permiten hacer visible la formación de representaciones de la sociedad desde las proyecciones de un deber ser vinculado a ciertos marcos valórico-normativos que movilizan actores concretos, y que recuperan para ello el eje temporal. El trabajo resulta interesante por como ilustra el uso de las categorías de análisis, así como por los resultados a los cuales llega.

Otros tres trabajos exponen la reflexión metodológica al servicio del análisis de la construcción de objetos. En el primero, "Los alcances de un diagnóstico comunitario sobre el suicidio: reflexiones a partir de una experiencia”, las autoras Castillo y Maroto articulan una reflexión sobre la propia experiencia en un 
diagnóstico comunitario referido al suicidio en una comunidad rural de San José, Costa Rica. En la primera parte describe las etapas involucradas en el diagnóstico relevando la necesidad de establecer lazos o vínculos con la población a través de su involucramiento en los distintos momentos de la investigación, y ubicando dicho diagnóstico desde un enfoque comunitario que sitúa al suicidio como un fenómeno social; estas reflexiones tempranamente fueron incorporadas por Emile Durkheim. Lo interesante del trabajo radica en el abordaje que presenta, en particular en relación con el modo de enfrentar de manera participativa una problemática de fuerte implicancia socioemocional, combinando cuestiones prescriptivas propias de diseños participativos o de intervención social con recomendaciones levantadas desde la experiencia del proyecto. Estos trabajos traen a la luz cuestiones de la trastienda que a menudo quedan solapados; la metodología cobra sentido cuando la experiencia y las prácticas de quienes han "recorrido el camino" nos ilustran y advierten respecto a hacer investigación en relación a determinadas temáticas.

El segundo analiza un libro llamado a constituirse en clásico del trabajo etnográfico: En busca de respeto. Vendiendo crack en Harlem. En el artículo “¿Cómo escribe el etnógrafo?”, Furaro presenta una reflexión sobre la formación del autor a través de una serie de estrategias y/o recursos que se evidencian en los propósitos y posicionamientos, así como en la utilización de ciertas conceptualizaciones que se urden en la narrativa o en el relato etnográfico. El artículo el trabajo de Bourgois utilizando fragmentos e incorporando categorías de análisis -autoridad, polifonía, dialogía- que se orientan desde lo que podríamos denominar una lectura de la escritura etnográfica como "texto" o como "genero discursivo", y que tiene como referente al "giro reflexivo" que vive la antropología americana hacia inicios de los '80. El artículo resulta una interesante reflexión sobre la metodología de Bourgois, si se piensa la metodología como la forma en que el autor fue construyendo el texto.

En el tercer y último artículo del volumen, Ana Safranoff presenta un análisis de cómo se define la categoría maltrato en un estudio de violencia de género que tiene como base la IV Macroencuesta de Violencia de Género de España (2011). Al distinguir los enfoques que operativizan la categoría maltrato -declarado y técnico- en el contexto de la violencia de género, la investigadora reflexiona sobre la interpretación indirecta- que realizan quienes responden, y que implica una aproximación a la dimensión semántica que está contendida en las preguntas. La encuesta antes referida permite este ejercicio al incluir preguntas asociadas a las dos conceptualizaciones de maltrato antes señaladas. Con fines analíticos se realiza una selección de casos y selección y transformación de las variables, a partir de los cuales se ponen en evidencia los efectos que generan uno u otro indicador, así como los "límites comprensivos" desde los cuales se interpreta la violencia por parte de las mujeres encuestadas, lo cual se encuentra, además, cruzado por la edad y nivel educacional.

El volumen concluye con una reseña al libro Redacción científica: cómo entender y escribir con facilidad, de Gonzaga Ferreira, presentado por Araujo Cavalcanti y Barbosa de Moura.

Los lectores podrán encontrar en el presente volumen trabajos que son expresión de la continua revisión que como investigadores sociales realizamos sobre nuestro quehacer.

\section{Notas}

1 En 2001 Charles Ragin publica Redesigning Social Inquiry. Fuzzy Sets and Beyond, que analiza la lógica de la investigación convencional introduciendo una serie de reflexiones muy interesantes orientadas desde el pensamiento difuso en torno al sentido de las correlaciones, N-Small y los casos, el análisis causal, la medición, la independencia, el análisis contrafactual, entre otros. 
$\underline{2}$ Agregar que cualquier interpretación se juega en la distinción entre lo verosímil e inverosímil. No todas las interpretaciones son posibles, y por ello el horizonte de posibilidad marca el límite entre interpretación y sobreinterpretación. Más allá de todas las variaciones o estilos, el concepto exige una continuidad rastreable entre lo primario y secundario a riesgo de transformarse en otra cosa. Un estilo es debido a que existe algo que une a pesar de su variación o exceso de sentido. En este sentido, al denominada autoetnografía, ¿es realmente un estilo etnográfico?, o, por otro lado, ¿̇es posible una etnografía virtual?; se pierde la continuidad estilística que implica como base la experiencia cotidiana y corpórea con un “otro”.

\section{Referencias}

Bourdieu, P. y Wacquant, L. (2005). Una invitación a la sociología reflexiva. Buenos Aires: Siglo XXI.

Freeman, L. (2004). The Development of Social Network Analysis. A Study in the Sociology of Science. Vacouver: Empirical Press.

Ragin, Ch. (1987). The Comparative Method. Moving Beyond Qualitative and Quantitative Strategies. California: Universidad de California Press.

Ricoeur, P. (2008). Hermenéutica y acción. De la hermenéutica del texto a la hermenéutica de la acción. Buenos Aires: Prometeo.

Sánchez Carrión, J. (2001). Estadística, orden natural y orden social. Revista Papers, 63/64, 33-46. 